\title{
Mapping quasifission characteristics in heavy element formation reactions
}

\author{
D.J. Hinde ${ }^{1, a}$, E. Williams ${ }^{1}$, R. du Rietz ${ }^{1, b}$, M. Dasgupta ${ }^{1}$, A. Wakhle ${ }^{1, c}$, C. Simenel ${ }^{1}$, D.H. Luong ${ }^{1}$, and K.J. Cook ${ }^{1}$ \\ ${ }^{1}$ Department of Nuclear Physics, RSPE, Australian National University, Acton, ACT 2601, Australia
}

\begin{abstract}
Mass-angle distributions carry detailed information on the characteristics of quasifission, and thus of the dynamics of heavy element formation reactions. Recent experimental results are presented and discussed.
\end{abstract}

\section{Introduction}

To form very heavy and superheavy elements (SHE), heavy-ion fusion reaction are used. Their cross sections can be significantly suppressed [1] by quasifission [2]. This dynamical non-equilibrium process results when the combined system formed after capture separates into two (fission-like) fragments in of order $10^{-20} \mathrm{~s}$, before a compact compound nucleus is formed. The probability of quasifission $\left(\mathrm{P}_{Q F}\right)$ can be very large, with the corresponding probability of compound nucleus formation $\left(\mathrm{P}_{C . N .}=1\right.$ $\mathrm{P}_{Q F}$ ) being lower than $10^{-3}$ in unfavourable cases. Understanding the competition between quasifission and fusion is thus very important in predicting the best fusion reactions to use to form new isotopes of heavy and super-heavy elements.

Because of the complex dependence of quasifission characteristics on many variables [3-6], and the overlap between quasifission and fusion-fission events in experiments, quasifission is not yet fully understood. As a wholly dynamical process, a key quantity characterizing quasifission is its timescale - that is the "sticking time" between capture and breakup (scission). Measurements of quasifission mass-angle distributions (MAD - described in detail below) at GSI in the 1980s [2, 7] showed that timescales could often be shorter than the rotation time of $\sim 10^{-20} \mathrm{~s}$, significantly less than the typical timescale of fusion fission. The measurement of MAD thus offers a key insight into the quasifission process. However, due partly to experimental difficulties, since the GSI work only a few measurements $[8,9]$ were made until recent years, when an extensive series of experiments (using the Australian National University Heavy Ion Accelerator Facility and CUBE spectrometer) has been carried out [3-6, 10-15]. The kinematic coincidence technique used in the measurements $[2,16]$ provides direct information on the mass-ratio of the fragments at scission. To avoid ambiguities in trans-

\footnotetext{
ae-mail: david.hinde@anu.edu.au

${ }^{\mathrm{b}}$ Current address: Malmö University, Faculty of Technology and Society, 20506 Malmö, Sweden

${ }^{\mathrm{c}}$ Current address: National Superconducting Cyclotron Laboratory, Michigan State University, East Lansing, Michigan 48824, USA
}

lation into fission fragment masses (i.e. scission masses with or without correction for pre-scission neutron evaporation, or final fission fragment masses after correction for post-scission evaporation as well), the data are all represented in terms of mass ratio $\mathrm{M}_{R}$. In this work, we have measured mass-angle distributions at many different beam energies, for many reactions forming heavy elements, using projectiles from $\mathrm{C}$ to $\mathrm{Ni}$ [6]. These have shown a rich spectrum of behaviour in quasifission.
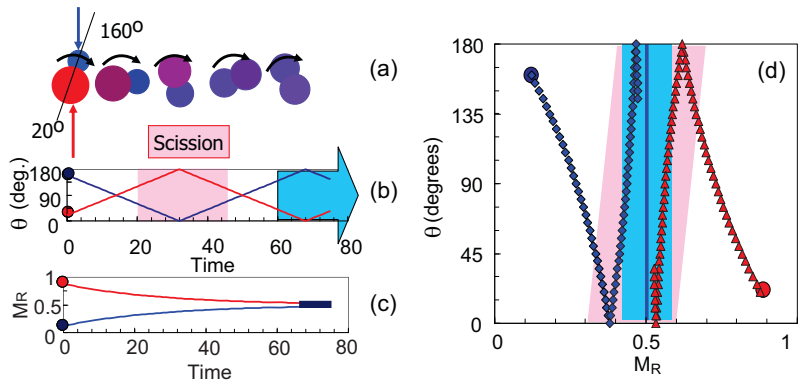

Figure 1. The relationship between sticking time and the massangle distribution. For the reaction sequence sketched in (a), the time dependence (arbitrary units) of angle (b) and mass-ratio (c) are shown. Such short times cannot be measured directly, but angle and mass-ratio can. The individual mass and angle dependencies combine to give a trajectory (d) on the MAD for a single impact parameter. Including a range of impact parameters, scission after half a turn (pink) or a full turn (blue) will give strong or weak mass-angle correlations respectively.

\section{Principle behind the mass-angle distribution}

The measurement of the full range of mass-splits between projectile and target over a wide range of scattering angles results in a two-dimensional matrix, referred to as a mass-angle distribution. The relationship of the MAD to the "sticking time" between capture and scission is illustrated schematically in Fig. 1. The projectile nucleus (blue) is incident from the top of the page, and sticks to 


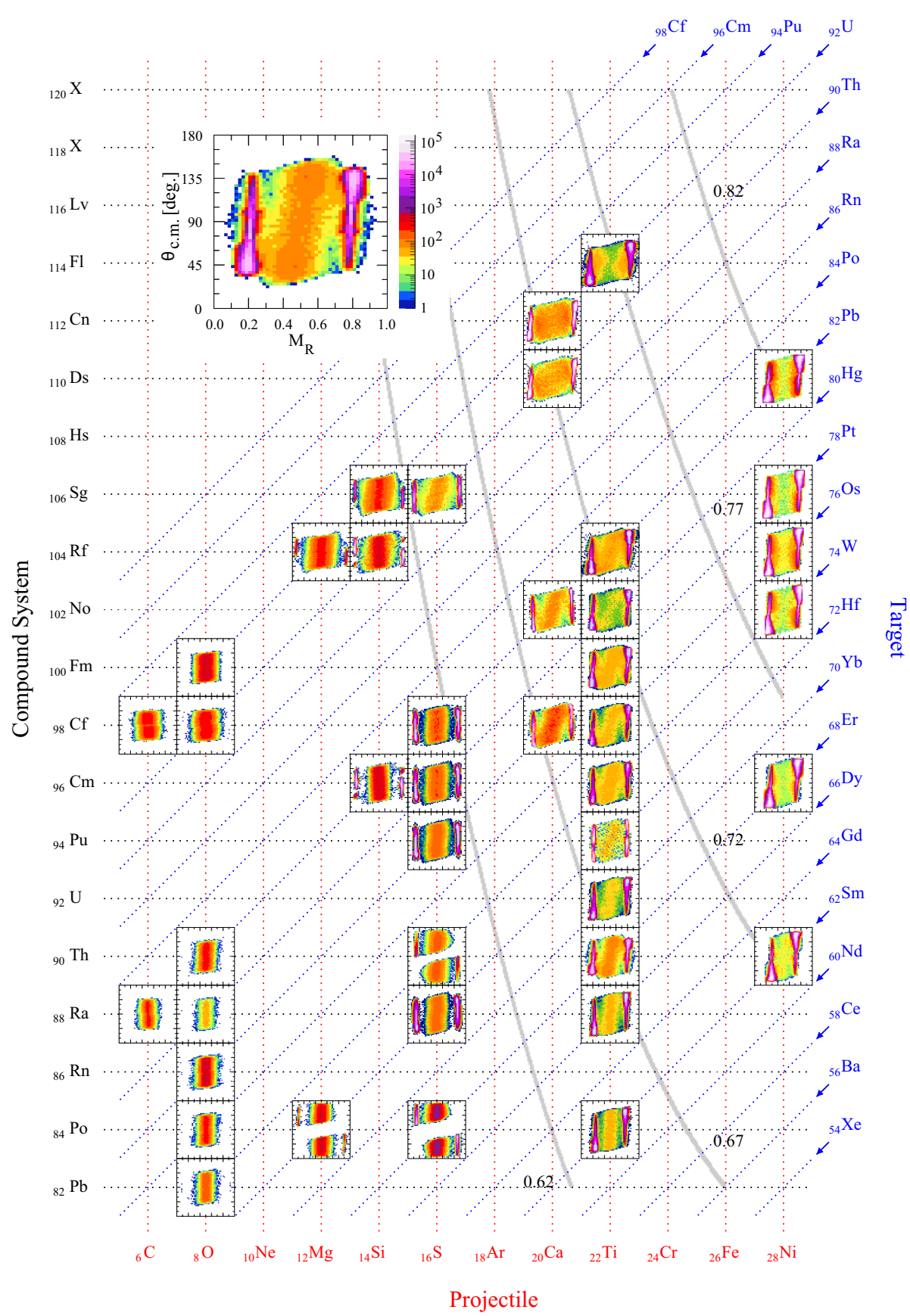

Figure 2. MAD scatter plots for all reactions studied. All miniature plots have the same axes as the large MAD example (for the $\mathrm{Ti}+\mathrm{Yb}$ reaction) at the top left. In the main plot, red vertical and blue diagonal dashed lines correspond to the same projectile and target atomic number, respectively, while the horizontal black dotted lines indicate the compound nucleus atomic number. The intensity scale represents counts per pixel in the MAD, proportional to $d^{2} \sigma / d \theta_{c . m} d M_{R}$. The full grey lines correspond to the indicated constant values of the entrance channel effective fissility (see text).

the larger target nucleus (red). The system then rotates, Fig. 1(b) illustrating schematically angle against time (in arbitrary units) for a single angular momentum value; in a real reaction a distribution will be present. For a parabolic potential, mass-symmetry is approached with an expected time dependence $1-\exp \left(\frac{t}{\tau_{e q}}\right)$, where $\tau_{e q}$ is the mass-equilibration time constant [2]. This dependence is sketched in Fig. 1(c).

If scission happens soon after initial contact, then little mass change can occur, and a projectile-like fragment is ejected with mass-ratio $M_{R}$ at backward angle $\theta_{c . m .} . M_{R}$ is defined as the mass of one fragment divided by the total mass involved in the collision. Its complementary binary fragmentation partner with mass-ratio $\left(1-M_{R}\right)$ is found at $\left(\pi-\theta_{c . m .}\right)$, a forward angle. A longer sticking time of the system results in larger rotation angles, and also allows more mass exchange. This evolution is illustrated on the MAD shown in Fig. 1(d). Rotation of the system by $\sim 180^{\circ}$ still results in a substantial mass-angle correlation (pale pink shading in Fig. 1(b),(d)), whilst once the system has 
turned $\sim 360^{\circ}$ or more, the correlation between the mass ratio and fragment emission angle is washed out, resulting in symmetric mass splits on average, independent of angle (blue shading).

\section{The MAD Map}

The approach we have taken to improve our quantitative understanding of the dynamics of quasifission finds an analogy with the liquid drop model approach to nuclear masses, in which localized shell effects can be quantified only when the underlying smooth trends are well defined. We have recently attempted to identify the broad trends of quasifission behaviour, an essential step to allow a quantitative exploration of the detailed dependence of quasifission on the nuclear structure of the colliding nuclei.

The approach begins by choosing measurements at beam energies somewhat above the capture barrier (typically by $\sim 6 \%$ ). Here the known effects of deformation alignment $[3,12,16,17]$ and shell structure observed in measurements at below-barrier energies $[4,18]$ are much reduced $[3,19,20]$. However the beam energies should not be too far above the capture barriers, otherwise high angular momenta would be introduced in the collisions. This is undesirable for two reasons. The first is empirical: the measurements should still be representative of the energies and angular momenta used in heavy element production reactions. The second is that for comparison with models aiming to predict $P_{C N}$, the mass-angle distributions should largely reflect the competition between the Coulomb and nuclear potentials, rather than being dominated by the repulsive angular-momentum dependent centrifugal potential.

The dependence of our measured mass-angle distributions on the atomic numbers of the projectile, target and compound nucleus are shown in Fig. 2. This "MAD Map" shows clear trends in the features of the MAD. The top right, corresponding to both heavy projectiles and heavy compound nuclei, shows a minimum in mass yield at symmetry, completely inconsistent with much contribution from fusion-fission, being rather closer to deepinelastic scattering. This category we term MAD1 in future discussions. The lower left (light projectiles and compound nuclei) shows distributions consistent with fusionfission, having a narrow mass distribution, and no significant correlation of mass and angle. This category is termed MAD3. Between these extremes, a smooth transition occurs, with MAD showing a strong but smoothly varying mass-angle correlation, typically consistent with "sticking" times corresponding to around half a turn of the composite system formed after capture. This class of MAD is termed MAD2.

\subsection{A quantitative measure of sticking times}

To explore quantitatively how different sticking times are reflected in changes in the MAD, a Monte Carlo simulation of mass-angle distributions for quasifission was developed [6]. This model, based on the physical picture pro-
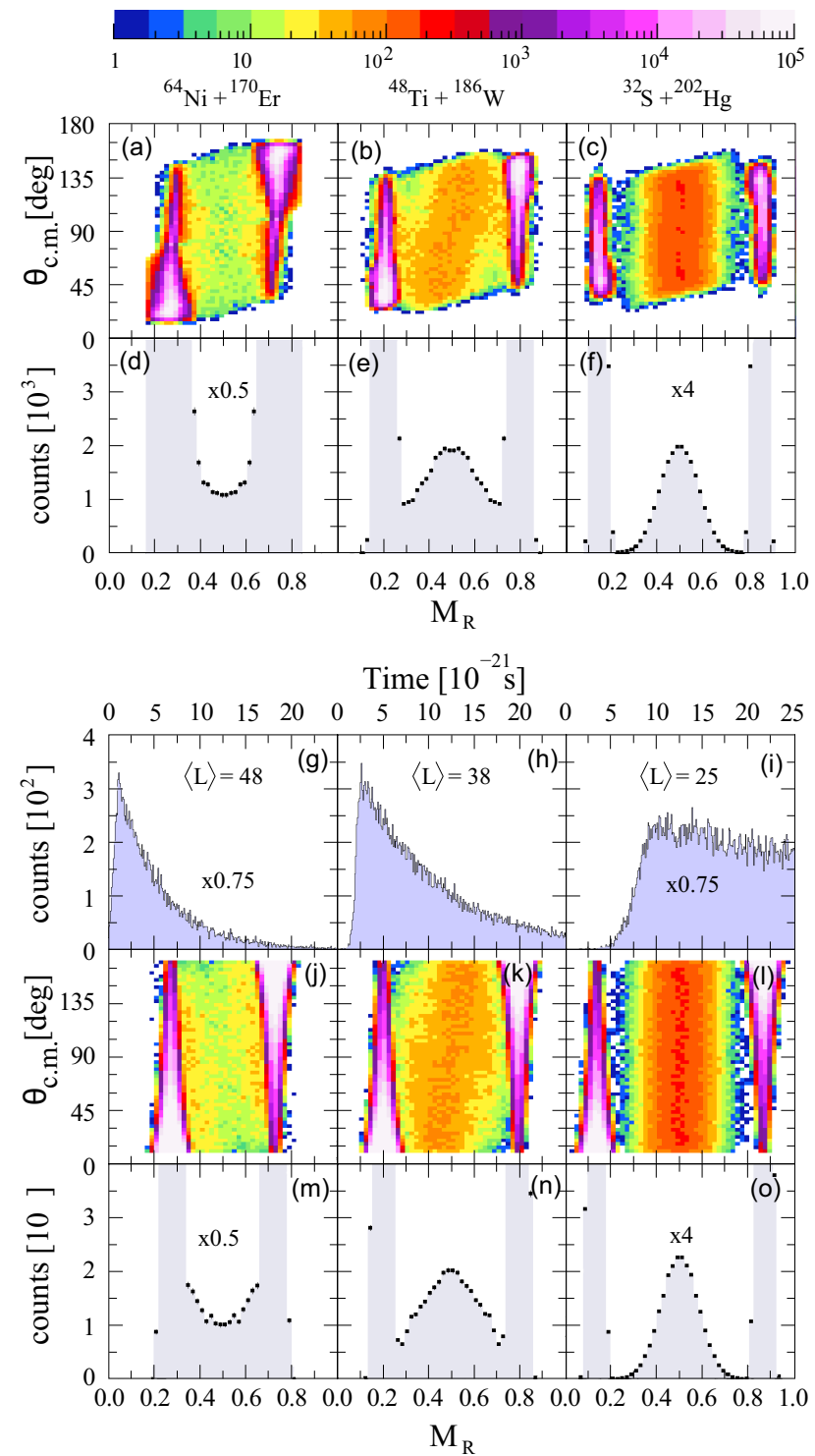

Figure 3. (Color online) The upper six panels show the experimental MAD in panels (a),(b),(c), and corresponding projections onto $M_{R}$ in panels (d),(e),(f), for the reactions ${ }^{64} \mathrm{Ni}+{ }^{170} \mathrm{Er}$, ${ }^{48} \mathrm{Ti}+{ }^{186} \mathrm{~W}$ and ${ }^{32} \mathrm{~S}+{ }^{202} \mathrm{Hg}$ (see text). The multiplicative factors shown scale the $y$-axis. The lower nine panels show simulated MAD for same reactions and energies in (g),(h),(i), with the corresponding $M_{R}$ spectra in panels $(\mathrm{m}),(\mathrm{n}),(\mathrm{o})$, and the sticking time distributions in $(\mathrm{j}),(\mathrm{k}),(\mathrm{l})$. These result in reasonable agreement between the simulations and the measurements shown in the top panels.

posed in Ref. [2], and first applied in Ref. [13], uses a simple description of mass equilibration and rotation to quantify how quasi-fission timescales determine the features of the MAD. Key inputs are the distribution of sticking times in the system, the time scale for mass equilibration [2], and the angular momentum distributions following fusion. The last ingredient is obtained from coupled-channels calculations performed using CCFULL [21]. Details of the model can be found in Ref. [6].

The simulation can only provide an estimate of angular velocities without input of time-dependent moments 
of inertia, thus it should not yet be used to obtain precise timescales. It can provide a comparative estimate of timescales for each of the three MAD classes. With this in mind, three reactions representing each of the MAD types have been chosen: ${ }^{64} \mathrm{Ni}+{ }^{170} \mathrm{Er}$ for MAD $1,{ }^{48} \mathrm{Ti}+{ }^{186} \mathrm{~W}$ for $\mathrm{MAD} 2$, and ${ }^{32} \mathrm{~S}+{ }^{202} \mathrm{Hg}$ for MAD3.

Both the observed and calculated MAD and mass-ratio distributions are shown in Fig. 3, with the observed quantities in panels (a) to (f) and the calculated quantities in panels ( $\mathrm{g}$ ) to (o). The distributions of sticking times used in each calculation are shown, as are the CCFULL calculated mean angular momenta for each reaction following capture.

For ${ }^{64} \mathrm{Ni}+{ }^{170} \mathrm{Er}$, the MAD is reproduced with a very short mean sticking time: $\tau_{\text {MAD1 }}<5 \cdot 10^{-21} \mathrm{~s}$. In the case of ${ }^{48} \mathrm{Ti}+{ }^{186} \mathrm{~W}$, a mean sticking time of $\tau_{\mathrm{MAD} 2} \sim 10 \cdot 10^{-21} \mathrm{~s}$ reproduces the experimental MAD. Finally, a lifetime corresponding to $\tau_{\mathrm{MAD} 3} \gg 10 \cdot 10^{-21} \mathrm{~s}$ provides a good match to the observed MAD for ${ }^{32} \mathrm{~S}+{ }^{202} \mathrm{Hg}$, having a very small fraction of fission before $10 \cdot 10^{-21} \mathrm{~s}$.

The sticking time distributions used to reproduce the observed MAD for ${ }^{64} \mathrm{Ni}+{ }^{170} \mathrm{Er},{ }^{48} \mathrm{Ti}+{ }^{186} \mathrm{~W}$ and ${ }^{32} \mathrm{~S}+{ }^{202} \mathrm{Hg}$ are consistent with those deduced for the three similarly categorized reactions presented in Ref. [13]. It is thus reasonable that these timescales should provide a representative measure of the reaction timescales for all reactions assigned to the three MAD classes.

Even where quasifission is dominant, there is the possibility of a fraction of late-chance fusion-fission events with lifetimes of $10^{-18}$ s or longer [22]. The MAD is quite insensitive to different time distributions for fission slower than $10 \cdot 10^{-21} \mathrm{~s}$. However, other experimental techniques are sensitive only to fission with very long time scales, such as the crystal blocking [23] and X-ray techniques [24] recently applied by Morjean and co-workers in superheavy element formation reactions. Clearly, the information on time scales from MAD and other techniques must be combined consistently to obtain a full picture of the distribution of reaction outcomes and times.

\section{Systematic trends of MAD characteristics}

The simple dependence of the MAD characteristics on the atomic numbers of the projectile and target nuclei seen in Fig. 2 suggests that it should be possible to linearize the trends in the MAD using one or two relatively simple ordering parameters.

\subsection{Ordering of MAD by charge product}

The simplest ordering, very similar to the axis variables of the MAD map in Fig. 2, is shown in Fig. 4. On the horizontal axis is the charge product $\mathrm{Z}_{p} \mathrm{Z}_{t}$, related to the Coulomb energy in the entrance channel. The vertical axis is the atomic number of the compound system $\mathrm{Z}_{C N}$, the most important variable determining the fissility of the compound nucleus. These axes allow any reaction to be very simply located on the plot, at the expense of neglecting the likely

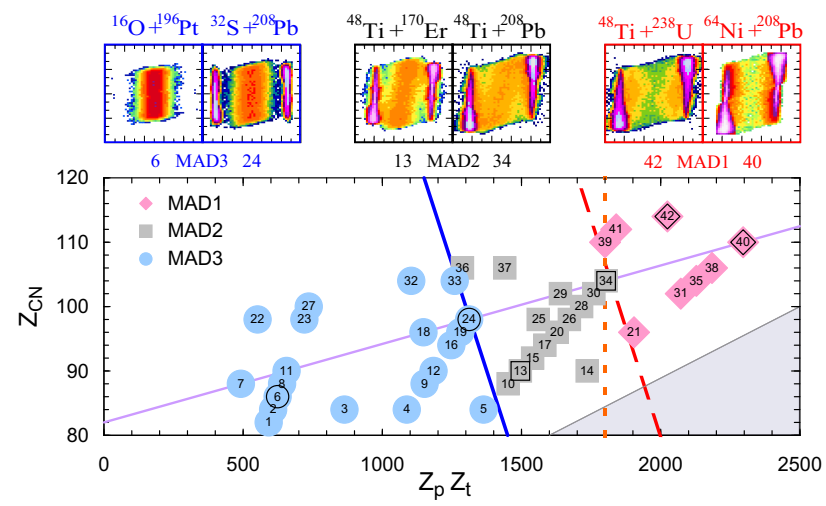

Figure 4. (Color online) The numbers in the figure refer to the reaction number in the Table of Ref. [6], plotted against entrancechannel charge product and compound atomic number. The colored symbol surrounding each number identifies the classification of the experimental MAD, as indicated in the legend at the lop left. The grey shaded region at the bottom right indicates where no binary reactions can form the compound nucleus. The thin purple line is the locus of reactions with $\mathrm{Pb}$. The diagonal full blue line represents the empirical boundary between reactions with no mass-angle correlation (left) and those that have (right). The dashed and dotted red lines indicate the uncertainty in the boundary of reactions which no longer exhibit a peak at symmetry in the angle-integrated fission mass distribution (see text). Examples of MAD are shown in the panels above, identified by their reaction number.

influence of the number of neutrons. Two examples from each MAD class are plotted in the upper panels. The reaction identification number of each of these examples is outlined in black on the graph below. In the grey shaded region in the lower right, no reactions are possible. The continuous thin purple line from bottom left to top right corresponds to reactions with isotopes of $\mathrm{Pb}$, whose magic numbers would be expected to influence the outcomes [4], if they were not attenuated by the selected higher excitation energies.

The different MAD classes cluster strongly, and approximate boundaries can be drawn (thick lines) between each class. The boundary where a mass-angle correlation becomes significant (between MAD classes 2 and 3) shows a dependence on both the variables in the graph. It is reasonable that this threshold should depend not only on the entrance channel, but also on the identity of the compound nucleus being formed, since long timescales imply the system reached much more compact shapes than the contact configuration. Thus the identity of the compound nucleus should play a role. The boundary for $Z_{C N} \sim 100$ $(\mathrm{Fm})$ occurs at $Z_{p} Z_{t} \sim 1300$, and assuming a linear dependence on $Z_{C N}$, the trend of the data is represented by the blue full line. This threshold shows a variation from $\mathrm{Z}_{p} \mathrm{Z}_{t}=1450 \pm 100$ at $\mathrm{Z}_{C N}=80$, down to $1150 \pm 100$ at $\mathrm{Z}_{C N}$ $=120$.

The location of the boundary between MAD classes 1 and 2 is not so well defined, because the density of data points is lower in this region. In particular, it is not clear whether the dividing line is dependent on $\mathrm{Z}_{C N}$ or not. The 
long dashed line uses the same gradient as the class 2 and 3 divider, whilst the short dashed line divides the data at $\mathrm{Z}_{p} \mathrm{Z}_{t}=1800$, independent of $\mathrm{Z}_{C N}$.

The projectile atomic numbers used in reactions with different target atomic numbers populate diagonal lines (with positive slope) in this representation. It is easy to distinguish lines within the figure corresponding to reactions with the same projectile element. For example, the band consisting of reaction numbers $14,21,31,35,38$, and 40 corresponds to reactions with ${ }_{28}^{64} \mathrm{Ni}$. Where the projectile band crosses the thick blue and dashed/dotted red lines in the figure, it indicates a change in the MAD class.

\subsection{Ordering of MAD by fissility parameters}

We now move to a mapping where the variables reflect the balance between nuclear and Coulomb forces during the collision. This balance is expected to determine the dynamics of reactions forming very heavy elements.

Ref. [25] discussed in detail the expectation of scaling behavior within the context of the schematic "chaotic regime dynamics" model of fusion of heavy nuclei. This is the model [26] that predicted the "extra push" and "extraextra-push" kinetic energies needed to overcome the conditional and unconditional saddle-point energies respectively. The successful scaling of calculated "extra-extrapush" energies, and the highlighting of associated landmark saddle-point configurations in the dynamical shape evolution, provides a background and motivation to attempt to order the experimental MAD outcomes in terms of: (i) the effective fissility parameter of the entrance channel, $x_{\text {eff }}$, and (ii) the fissility parameter of the compound nucleus, $x_{\mathrm{CN}}$. Unlike the atomic numbers used in previous mapping, each variable has a quantitative interpretation [25], being a measure of the balance between the repulsive Coulomb force and the attractive nuclear force. The former applies to necked shapes close to the (generally) mass-asymmetric contact configuration in the entrance channel, whilst the latter applies to shapes without a constricted neck [26], and where the mass-asymmetry degree of freedom is not constrained.

Using the same format as Fig. 4, the data are plotted in Fig. 5(a) against these two fissility parameters. In this representation the mass numbers (neutron numbers) of the colliding nuclei play a role in calculating both fissility parameters, unlike in the previous mappings. However, as might be expected from the smaller effect of neutron number compared to proton number in determining fissilities, the data still cluster in the three classes in a similar way to that seen in Fig. 4.

The boundary across which a mass-angle correlation becomes significant (between MAD classes 3 and 2) again shows a dependence on both variables, with a stronger dependence on the entrance-channel than on the compound nucleus fissility. The full blue line is our estimate of this boundary based on the current data. Mass-angle distributions for reactions on this line should show similar massangle correlations, associated with similar reaction trajectories and timescales. The same should be true for reac-
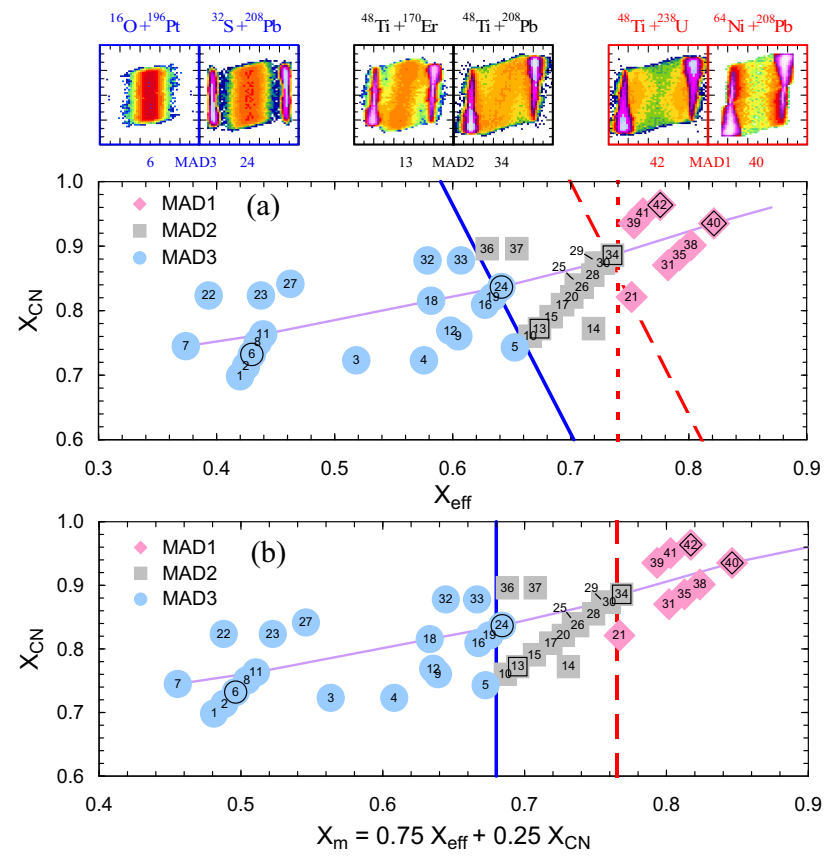

Figure 5. (Color online) As in Fig. 4, but here plotted (a) as a function of the effective fissility parameter in the entrance channel $x_{\text {eff }}$ and the compound nucleus fissility parameter, and (b) as a function of the mean fissility parameter $\mathrm{x}_{m}$ required to straighten the MAD class boundaries (see text), and the compound nucleus fissility parameter.

tions on nearby parallel lines. The equation of this boundary line is $0.75 x_{\text {eff }}+0.25 x_{\mathrm{CN}}=0.68$, giving $x_{\text {eff }}$ three times the weight of $x_{C N}$. In terms of the MAD, this defines a mean fissility parameter $\mathrm{x}_{m}$ which seems to give a good characterization of the quasifission observed, at the higher beam and excitation energies chosen here.

\section{Conclusions}

Having taken the first steps to defining a smooth "liquid drop" behaviour, deviations relating the magic numbers and static deformation can more easily be quantified. From an experimental perspective, several steps still have to be taken to realise the full potential of MADs in probing quasifission. These include:

- experimental isolation of the effect of angular momentum on time scales;

- experimental confirmation of the mass-equilibration time constant;

- investigation of the role of neutron richness in quasifission dynamics independent of shell effects;

- more extensive MAD measurements to study the effects of magic numbers at and below the capture barrier energy;

- extension of measurements into the angular region currently with no coverage, to obtain total mass yields.

These questions should be addressed in future MAD measurements. Refinement of the Monte Carlo MAD sim- 
ulation model should help to address the first two questions, but the ultimate goal is that sophisticated dynamical models should calculate MADs directly, which can then be compared quantitatively with the corresponding experimental MADs. Quantum mean-field models such as timedependent Hartree-Fock [27-29] and extensions can make a significant contribution in developing reliable predictive power of quasifission and fusion probabilities [30, 31] in reactions forming new very heavy and superheavy elements and isotopes, including mapping out prospects of using neutron-rich radioactive beams.

\section{Acknowledgments}

The authors acknowledge outstanding support from Dr. $\mathrm{N}$ Lobanov and the ANU Heavy Ion Accelerator Facility staff, without whose tireless commitment to excellence this work would not have been possible, and financial support from ARC grants DP110102858, DP130101569, DP140101337, FL110100098, FT120100760 and DE140100784.

\section{References}

[1] D.J. Hinde, M. Dasgupta, A. Mukherjee, Phys. Rev. Lett. 89, 282701 (2002)

[2] J. Tōke, B. Bock, G.X. Dai, A. Gobbi, S. Gralla, K.D. Hildenbrand, J. Kuzminski, W. Müller, A. Olmi, H. Stelzer, Nucl. Phys. A 440, 327 (1985)

[3] D.J. Hinde, R.G. Thomas, R. du Rietz, A. DiazTorres, M. Dasgupta, M.L. Brown, M. Evers, L.R. Gasques, R. Rafiei, M.D. Rodriguez, Phys. Rev. Lett. 100, 202701 (2008)

[4] C. Simenel, D.J. Hinde, R. du Rietz, M. Dasgupta, M. Evers, C.J. Lin, D.H. Luong, A. Wakhle, Phys. Lett. B 710, 607 (2012)

[5] E. Williams, D.J. Hinde, M. Dasgupta, R. du Rietz, I.P. Carter, M. Evers, D.H. Luong, S.D. McNeil, D.C. Rafferty, K. Ramachandran et al., Phys. Rev. C 88, 034611 (2013)

[6] R. du Rietz, E. Williams, D.J. Hinde, M. Dasgupta, M. Evers, C.J. Lin, D.H. Luong, C. Simenel, A. Wakhle, Phys. Rev. C 88, 054618 (2013)

[7] W.Q. Shen, J. Albinski, A. Gobbi, S. Gralla, K.D. Hildenbrand, N. Herrmann, J. Kuzminski, W.F.J. Müller, H. Stelzer, J. Tōke et al., Phys. Rev. C 36, 115 (1987)

[8] J. Velkovska, C.R. Morton, R.L. McGrath, P. Chung, I. Diószegi, Phys. Rev. C 59, 1506 (1999)

[9] B.B. Back, P.B. Fernandez, B.G. Glagola, D. Henderson, S. Kaufman, J.G. Keller, S.J. Sanders, F. Videbæk, T.F. Wang, B.D. Wilkins, Phys. Rev. C 53, 1734 (1996)

[10] R.G. Thomas, D.J. Hinde, D. Duniec, F. Zenke, M. Dasgupta, M.L. Brown, M. Evers, L.R. Gasques, M.D. Rodriguez, A. Diaz-Torres, Phys. Rev. C 77, 034610 (2008)
[11] R. Rafiei, R.G. Thomas, D.J. Hinde, M. Dasgupta, C.R. Morton, L.R. Gasques, M.L. Brown, M.D. Rodriguez, Phys. Rev. C 77, 024606 (2008)

[12] D.J. Hinde, R. du Rietz, M. Dasgupta, R.G. Thomas, L.R. Gasques, Phys. Rev. Lett. 101, 092701 (2008)

[13] R. du Rietz, D.J. Hinde, M. Dasgupta, R.G. Thomas, L.R. Gasques, M. Evers, N. Lobanov, A. Wakhle, Phys. Rev. Lett. 106, 052701 (2011)

[14] C.J. Lin, R. du Rietz, D.J. Hinde, M. Dasgupta, R.G. Thomas, M.L. Brown, M. Evers, L.R. Gasques, M.D. Rodriguez, Phys. Rev. C 85, 014611 (2012)

[15] A. Wakhle, C. Simenel, D.J. Hinde, M. Dasgupta, M. Evers, D.H. Luong, R. du Rietz, E. Williams, Phys. Rev. Lett. 113, 182502 (2014)

[16] D.J. Hinde, M. Dasgupta, J.R. Leigh, J.C. Mein, C.R. Morton, J.O. Newton, H. Timmers, Phys. Rev. C 53, 1290 (1996)

[17] D.J. Hinde, M. Dasgupta, J.R. Leigh, J.P. Lestone, J.C. Mein, C.R. Morton, J.O. Newton, H. Timmers, Phys. Rev. Lett. 74, 1295 (1995)

[18] E.V. Prokhorova, A.A. Bogachev, M.G. Itkis, I.M. Itkis, G. Knyazheva, N.A. Kondratiev, E.M. Kozulin, L. Krupa, Y.T. Oganessian, I.V. Pokrovsky et al., Nucl. Phys. A 802, 45 (2008)

[19] K. Nishio, H. Ikezoe, S. Mitsuoka, I. Nishinaka, Y. Nagame, Y. Watanabe, T. Ohtsuki, K. Hirose, S. Hofmann, Phys. Rev. C 77, 064607 (2008)

[20] I.M. Itkis, E.M. Kozulin, M.G. Itkis, G.N. Knyazheva, A.A. Bogachev, E.V. Chernysheva, L. Krupa, Y.T. Oganessian, V.I. Zagrebaev, A.Y. Rusanov et al., Phys. Rev. C 83, 064613 (2011)

[21] K. Hagino, N. Rowley, A.T. Kruppa, Comp. Phys. Commun. 129, 143 (1999)

[22] Morjean, M., Chbihi, A., Dasgupta, M., Drouart, A., Frankland, J.D., Fregeau, J.D., Hinde, D.J., Jacquet, D., Nalpas, L., Parlog, M. et al., EPJ Web of Conferences 63, 02011 (2013)

[23] M. Morjean, D. Jacquet, J.L. Charvet, A. L'Hoir, M. Laget, M. Parlog, A. Chbihi, M. Chevallier, C. Cohen, D. Dauvergne et al., Phys. Rev. Lett. 101, 072701 (2008)

[24] M.O. Frégeau, D. Jacquet, M. Morjean, E. Bonnet, A. Chbihi, J.D. Frankland, M.F. Rivet, L. TassanGot, F. Dechery, A. Drouart et al., Phys. Rev. Lett. 108, 122701 (2012)

[25] J. Blocki, H. Feldmeier, W. Swiatecki, Nucl. Phys. A 459, 145 (1996)

[26] S. Bjornholm, W. Swiatecki, Nucl. Phys. A 391, 471 (1982)

[27] D.J. Kedziora, C. Simenel, Phys. Rev. C 81, 044613 (2010)

[28] C. Simenel, EPJ A 48, 441 (2012)

[29] V.E. Oberacker, A.S. Umar, C. Simenel, Phys. Rev. C 90, 054605 (2014)

[30] K. Washiyama, Contribution to FUSION 2014 proceedings, in EPJ Web of Conferences (2014)

[31] Guo, L., Nakatsukasa, T., EPJ Web of Conferences 38, 09003 (2012) 